\title{
Based on LabVIEW Secondary Power Station Program Design Zhao Chong ${ }^{1, a}$ and Ning Wang ${ }^{2, b}$ \\ ${ }^{1,2}$ Xijing University, Shaanxi Xi'an,710123,China \\ âE-mail:2741448598@qq.com, ${ }^{b}$ E-mail:3738242415@qq.com,
}

Keywords: Program; Encapsulation; VI

\begin{abstract}
Because of the PC program code is more, and part of the program has been repeated calls, in order to simplify the code, will be part of the code encapsulation as sub-VI, which makes the program more clear and concise structure, promotes the reuse of code, easy to maintenance and writing of the program. The following sub-vi: Agilent 34401 parameter set sub-VI, Tektronix TDS200 parameter Settings sub-VI, delay setting sub-VI, port configuration sub-VI, test program sub-VI.
\end{abstract}

\section{Introduction}

LabVIEW is a $\mathrm{G}$ language-based software development environment developed by National Instruments. Compared with standard laboratory instrument, LabVIEW function more powerful, at the same time have the flexibility, can be widely used in industrial control, physics research, agricultural production, laboratory teaching, psychological research, etc. This article USES LabVIEW to complete the design of the upper machine program for the test software system.

\section{Initialize the Parameter Settings}

In the main program of upper machine based on LabVIEW program before you begin, need for digital multimeter, digital oscilloscope, communication port and delay time DengZi VI initialization parameter is set, the following four sub VI provides program.

\section{Agilent 34401 Parameter Settings}

The Agilent 34401 parameter setup diagram is shown in Fig.1. The Agilent 34401 parameter setting dialog is shown in Fig. 2.

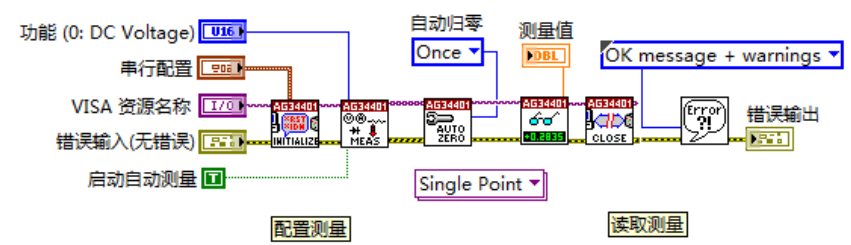

Figure 1 Agilent 34401 parameter sets the block diagram

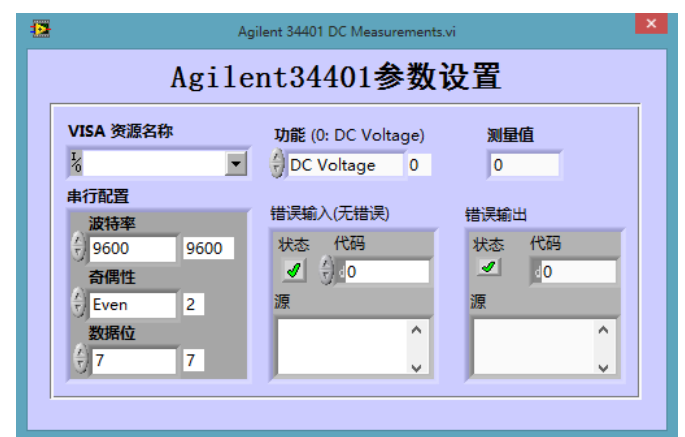

Figure 2 Agilent 34401 parameter sets the dialog interface

The subvi is used to implement the configuration of the parameters for the digital multimeter Agilent 34401 and the reading of the measurements. Using the driver and operating function nodes provided in LabVIEW, the digital multimeter Agilent 34401 is performed directly. 


\section{Tektronix TDS200 Parameter Settings}

The Tektronix TDS200 parameter setting program box is shown in Fig. 3. The Tektronix TDS200 parameter setting dialog looks like Fig.4.

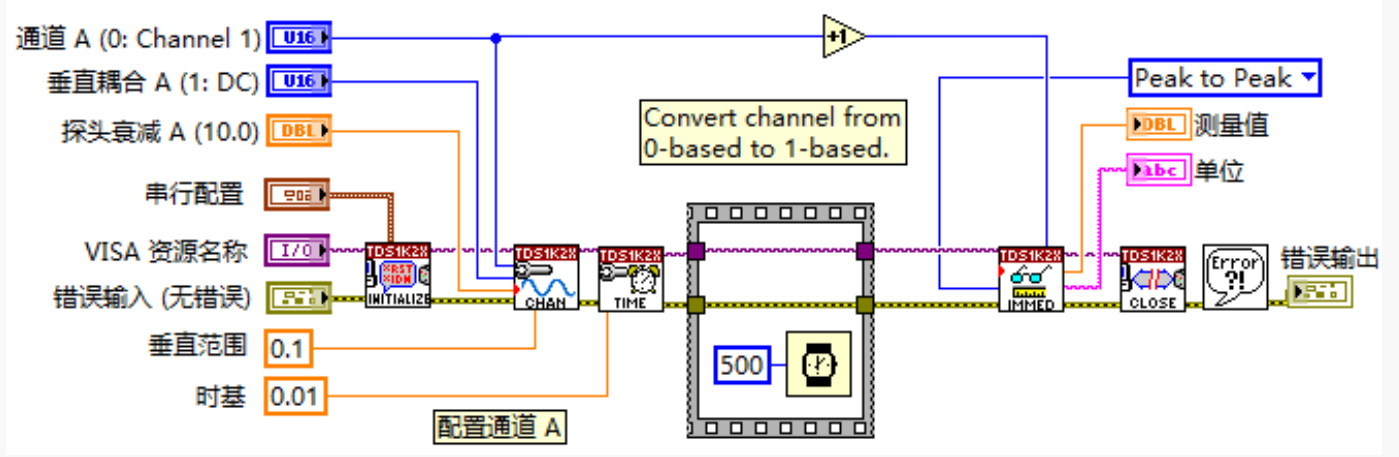

Figure 3 Tektronix TDS200 parameter sets the program block diagram

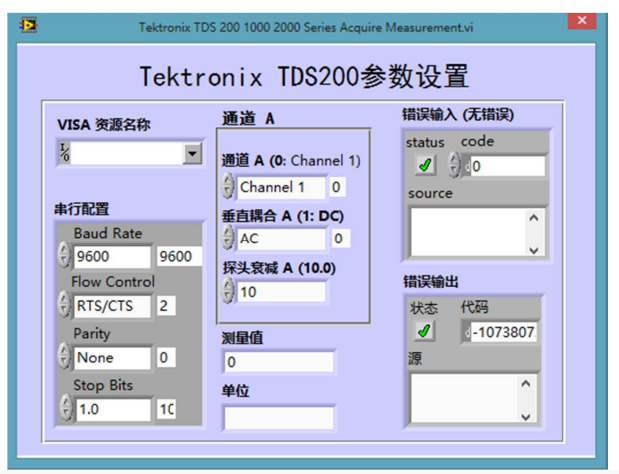

Figure 4 Tektronix TDS200 parameter sets the dialog interface

The subvi is used to implement the configuration of the parameters for the digital oscilloscope Tektronix TDS200 and the reading of the measurements. Similar to digital multimeter Agilent 34401 parameter Settings, from Tektronix TDS200 parameter setup program block diagram you can see, except for error handling "error in" and "error out", also called the digital oscilloscope Tektronix a few TDS200 operation node.

\section{Delay Setting}

The delay setting up the block diagram and the dialog screen is shown in Fig.5. The subvi takes the conditional structure and sets the delay time in milliseconds with the default branch of "no error". The front panel of the delay setting can determine whether or not the wait time is modified as needed.
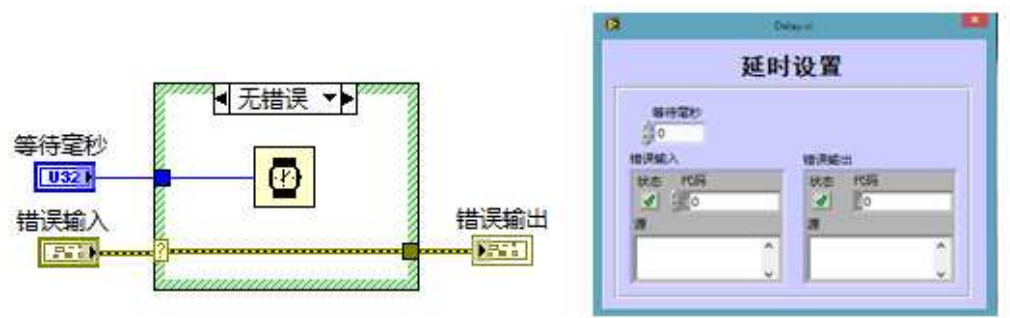

Figure 5. The time delay setting up the program block diagram and the dialog interface

\section{Port configuration}

The port configuration block diagram and the dialog screen are shown in Fig.6. In accordance with the design of the preceding article, the serial communication is carried out by using the common RS232 method between the peripherals and the computer. Before serial communication, call the configuration of the LabVIEW serial node to a serial port configuration, complete digital multimeter, digital oscilloscope and load cases of the three port after setting communication, between SCM and PC can realize the data communication. 

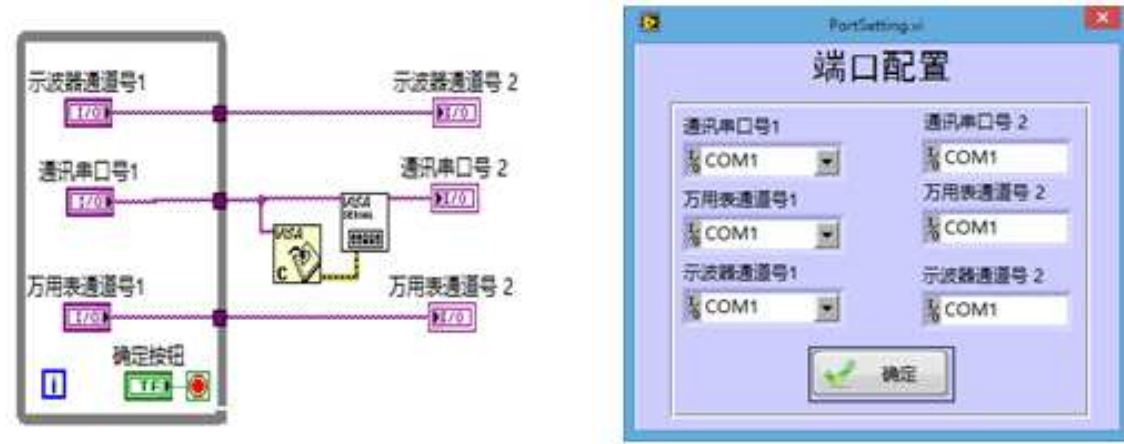

Figure 6 port configuration diagram and dialog interface

\section{Test Programming}

The test program block diagram is shown in Fig.7. The test procedure preferences dialog is shown in Fig. 8. For loop structure is used in outer parameters in the test program set up, the front panel can be done For the selection of load state, using three communication serial port to load cases of interconnection, multimeter and oscilloscope and the upper machine. The inner layer adopts the conditional structure and avoids the interference of the program function. First, the number and load conditions of the power supply are selected by the array. By "VISA again write function" write data, call delay subvi set data acquisition interval, called after multimeter subvi read voltage and current data, call the oscilloscope subvi read ripple data; Finally, the data is converted to a string after the various connections.

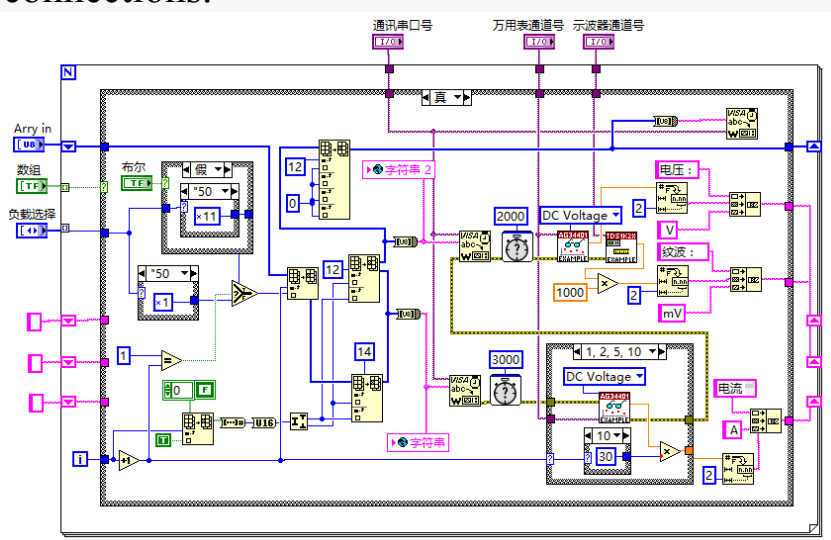

Figure 7 test program block diagram

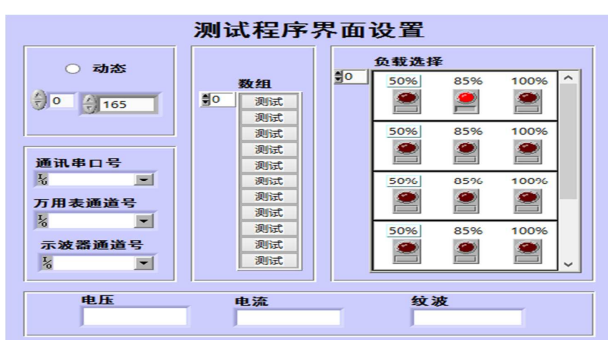

Figure 8 test procedure parameter sets the dialog interface

Wrap the test program in subvi for the convenience of the main interface. At this point, the subvi functionality of the automated test system is fully implemented.

\section{Operation Main Interface Design}




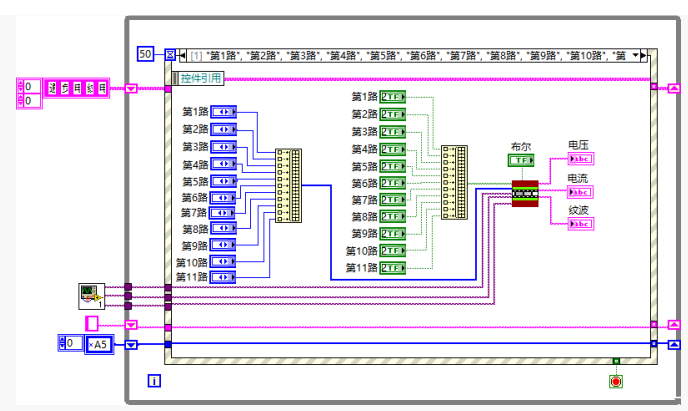

Figure 9 controls the flow diagram of the main interface of the computer

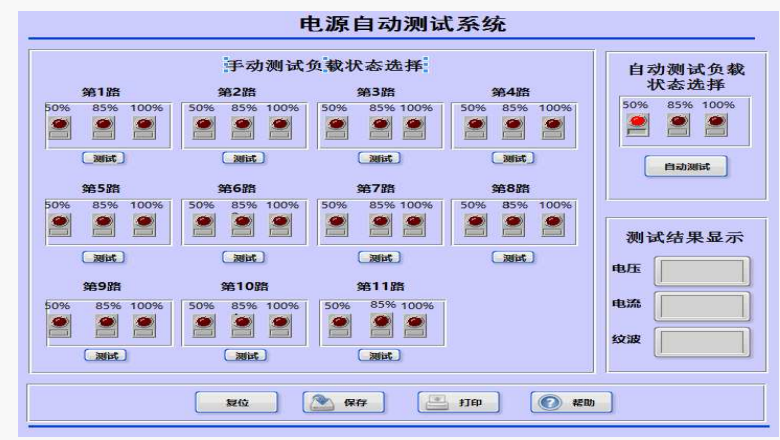

Figure 10 controls the main interface of the computer

The program block diagram for the main computer operating master interface is shown in Fig.9. The host interface is shown in Fig.10. This interface can automatically complete the data collection and data processing of the next machine and data store.

The yellow button below is the reset button, and when the test is implemented, it is reset.

As shown in Fig.10 in the left side of the screen provide 11 "test" option in the road respectively, can undertake single way of manual testing, testing road 3, for example, click the "test" button, "50\%", "85\%" and "100\%" three successive test lamp light, light red said is testing items, otherwise for or to be measured.

\section{Summary}

This paper designs the software solution of automatic test system. First, the overall scheme of software system design is presented, which is designed to be a three-part software monitoring software, lower machine monitoring software and MSP430 software. Second, the main program of MSP430 and the design process of single road subroutine are described. Then, the MSP430 and the previous communication programming method were described. Finally, from the initialization parameter is set, the test program design and the main operation interface design from three aspects, in detail introduced the PC program design based on LabVIEW, and each dialog box and program flowchart is presented.

\section{References}

[1]Alessandro Ferrero, Simona Salicone. A calibration procedure for a digital instrument for electric power quality measurement. IEEE Transactions on Instrumentation and Measurement, VOL. 51, NO. 4, AUGUST 2002.

[2] Zheng Dong, Dagui Huang, Deyin Zhang, Wenrong Wu.An Automatic MEMS Testing System based on Computer Microvision. Proceedings of the 2006 IEEE

[3] D. Wang, C. Mao, J. Lu, J. He, and H. Liu, "Auto-balancing transformer based on power electronics," Electric Power Systems Research, vol. 80, pp. 28-36, 2010. 
[4] Wang Pinglian, Yuan Sumin. Automated Measurement System for Wireless Transmitters. ICEMI 8th Electronic Measurement and Instruments International Conference, 2007.

[5] H. Iman-Eini, Sh. Farhangi, J.L. Schanen, and M. Khakbazan-Fard, "A modular power electronic transformer based on a cascaded H-bridge multilevel converter," Electric Power Systems Research, vol. 79, pp. 1625-1637, 2009. 